Revista Calidad en la Educación Superior

Programa de Autoevaluación Académica

Universidad Estatal a Distancia, Costa Rica

ISSN 1659-4703

revistacalidad@uned.ac.cr

\title{
EXPERIENCIA E INTERACCIÓN DE AULA QUE SE GENERAN COMO AMBIENTE DE APRENDIZAJE DURANTE LAS CLASES DE BIOLOGÍA.
}

\author{
EXPERIENCE AND CLASSROOM INTERACTION OF GENERATED AS CLIMATE \\ FOR LEARNING DURING BIOLOGY CLASSES.
}

José Pereira-Chaves ${ }^{1}$ jose.pereira.chaves@una.cr Universidad Nacional de Costa Rica Universidad Estatal a Distancia, Costa Rica

\author{
Volumen 7, Número 2 \\ Noviembre, 2016 \\ pp. $49-75$
}

Recibido: 18 de marzo, 2016

Aprobado: 15 de octubre, 2016

\footnotetext{
${ }^{1}$ Doctor en educación, Máster en Ciencias Marinas, Bachillerato y Licenciatura en Enseñanza de las Ciencias. Correo electrónico: jose.pereira.chaves@una.cr/ jpereira@uned.ac.cr
} 


\title{
Resumen
}

Las acciones que se genera en el aula como ambiente de aprendizaje son fundamentales para propiciar sinergias de trabajo armónico y de interés entre docentes y estudiantado. Las cuales deberán tener como finalidad un aprendizaje motivante de la disciplina. El objetivo de este trabajo fue evidenciar de manera discreta y preliminar, los tipos de relaciones-interacciones que se desarrollan en las lecciones de biología en un colegio secundario de la ciudad de Heredia de Costa Rica y cómo contribuyen a la construcción y comprensión del conocimiento que se promueve en el programa del Ministerio de Educación de Costa Rica. Se trabajó mediante un estudio de caso, aplicando la técnica de observación participante en aula(OP), entrevistas a estudiantes(EE), docente(EP) y un grupo focal(GF). Entre los resultados se destaca que la ecología de aula (relaciones sociales, afectivas y emocionales que se desarrollan durante la interacción) favorece el interés hacia el aprendizaje por parte de los estudiantes. Se discute acerca de la valoración de la reflexión teórica sobre la ciencia y su significado en el mundo, no sólo para favorecer ambientes que potencien el aprendizaje, sino que junto a ello, para que docentes y estudiantes desarrollen competencias de pensamiento científico que les permita 'leer el mundo' y valorar el conocimiento.

Palabras clave: Estudiantes, profesora, interacción, aula, aprendizaje.

\begin{abstract}
The actions generated in the classroom and learning environment sciences are essential to promote harmonious synergies of work and interest among teachers and students. They should aim a motivating learning scientific discipline. The objective of this work was to demonstrate discrete and preliminarily, the types of relationsinteractions that take place in biology lessons in a secondary school in the city of San Jose, Costa Rica and how they contribute to building knowledge and understanding It promoted in the program of the Ministry of Education of Costa Rica. We worked through a case study, applying the technique of participant observation in the classroom (OP), interviews with students (EE), teacher (EP) and a focus group (GF).Among the results emerged that ecology classroom ( social, affective and emotional relationships that develop during the interaction) promotes the interest towards learning by students. We discussed the assessment of theoretical reflection on science and its meaning in the world, not only to promote environments that enhance learning, but next to it, so that teachers and students develop skills of scientific thinking that allows them to 'read the world 'knowledge and value.
\end{abstract}

Keywords: Students, teacher interaction, classroom learning. 
El conocer la realidad educativa del aula, la dinámica de interacción entre el docente y los estudiantes, el contexto y el ambiente donde se desarrollan los conceptos biológicos, son elementos que favorecen la comprensión de las prácticas pedagógicas que se requieren para promover la formación de personas analíticas, creativas, autónomas, consientes y formadas con habilidades científicas de las diferentes realidades.

Motivar al estudiantado promueve la reflexión de los aspectos, hechos, situaciones y conceptos que deberán aprender entorno a los diversos fenómenos científicos y cotidianos, sin dejar de considerar que los factores que determinan la motivación en el aula habitualmente se darán a través de la interacción que se lleven a cabo en el interior de la misma.

Todas las actividades que se desarrollan en el salón de clases, deben estar orientadas con un alto sentido de pertinencia para los estudiantes, donde se incentiven inquietudes de conocimiento vinculadas a la comprensión de conceptos y fenómenos científicos significativos, así se captará el interés de los estudiantes y se trabajan diversas formas de aprendizaje, el tratamiento que se le dé en la mediación pedagógica es fundamental para vincular actividades que promuevan la comprensión del estudiantado en relación a los procesos biológicos reales por medio de sus interacciones en la ecología del aula.

Se considera la forma en que la docente se desempeña en el aula y la interacción de los estudiantes, tomando en cuenta la comunicación que facilite la construcción de conceptos. Se abordó cómo recibe el estudiante la información facilitada por el docente, su actitud, interacción y comunicación en aras de construir los conceptos biológicos. Así las interacciones, comprenden los procesos de asociación de unos actores conscientes con otros, entre los que se produce un intercambio, una orientación y una afectación de la conducta de unas personas con respecto a las demás, y con las cuales se establece una relación determinada. Estos procesos de 
interacción entre los miembros de un grupo específico generan una red de relaciones edificadoras de organización social y cultural (Blandón, Molina y Vergara, 2006, p. 14). Considerando las interacciones que se generan en el aula el objetivo de este trabajo fue evidenciar la realidad educativa que se lleva a cabo en un aula durante las clases de biología como elemento importante para la construcción del conocimiento científicobiológico.

\section{Aspectos Teóricos}

Entender los procesos de enseñanza y aprendizaje en el salón de clase, son puntos básicos y fundamentales que se requieren durante la mediación pedagógica de las ciencias de la vida y conjuntamente a ello las relaciones que se llevan a cabo en el aula. Hilera y Palomar (2008) señalan que el diseño de un proceso de enseñanza y aprendizaje es una tarea que todo educador tiene que realizar cuando ha de elaborar la forma de desarrollar el contenido. Es aquí cuando se deben plantear y considerar aspectos fundamentales en el proceso de socialización e interacción, como el contexto en el que se va llevar a cabo la docencia, el método más adecuado en ese contexto, los recursos didácticos necesarios, los contenidos de la actividad desde las diversas dimensiones, así como los criterios de evaluación a considerar para determinar si se han alcanzado los objetivos de aprendizaje previstos, lo que genera una ambiente asertivo y de cordialidad entre docente y estudiantes.

Además, partiendo de la visión sistémica, Estrada y Monroy (2002), reconocen que la realidad tangible y la realidad intangible no están divididas, ni ordenadas, ni organizadas, como casi siempre se suele considerarlas divididas. La realidad no está dividida en las clases, ni en las categorías que el humano ha ido estableciendo sobre la base del crecimiento del conocimiento, las experiencias y las características culturales propias. Por ello hablar de la enseñanza de la Biología es abordarla desde el enfoque particular de la disciplina, su naturaleza y complejidad de interacciones, donde se aporten análisis de las diferentes disciplinas que favorezcan la educación y la promoción de competencias del pensamiento científico y la búsqueda del conocimiento. 
El estudiante debe comprender y poner en práctica sus competencias y así socializar con sus compañeros aportes relevantes que permita poner en común intereses la construcción colectiva de nuevos conocimientos.

Por otra parte es fundamental destacar elementos importantes que se generan en los procesos de interacción en el aula, y eso influye en el estado anímico del individuo, lo cual es elemental articularlo como docente, durante el abordaje del contenido, considerando que la inteligencia emocional es fundamental e indispensable en el acto educativo, porque en todo proceso de interacción social alumnos-alumnos-profesor se ven inmersos una serie de elementos que dan el inicio del cumplimiento de los objetivos de aprendizaje, como las competencias afectivas y emocionales, así estas habilidades deben de ser consideradas lo cual lo expresa en su estudio, Extremera y Fernández (2004a) quienes señalan que las aulas son el modelo de aprendizaje socioemocional de mayor importancia para los estudiantes y a la vez porque los niveles de inteligencia emocional permiten que en el contexto educativo se manejen las presiones de una mejor manera.

Por otra parte Bisquerra et al. (2009) señalan que las competencias emocionales son parte de la alfabetización emocional, lo que radica en la capacidad de tomar conciencia emocional con efectos positivos sobre el profesorado y el alumnado por igual, aspecto que implica poner en prácticas dichas competencias. Así la capacidad que tenemos para manejar las emociones, actitudes, impulsos, valores, habilidades y capacidades propias del individuo favorece a que mediante el acto educativo se proyecte confianza, creatividad, y motivación en los aprendices, considerando el socioaprendizaje como elemento común en los actores de interés.

Simmons y Simmos (1998) señalan 13 escalas de inteligencia emocional que se muestran en la siguiente figura. 
Figura 1. Escala de inteligencia emocional basada en la propuesta de Simmons y Simmos (1998)

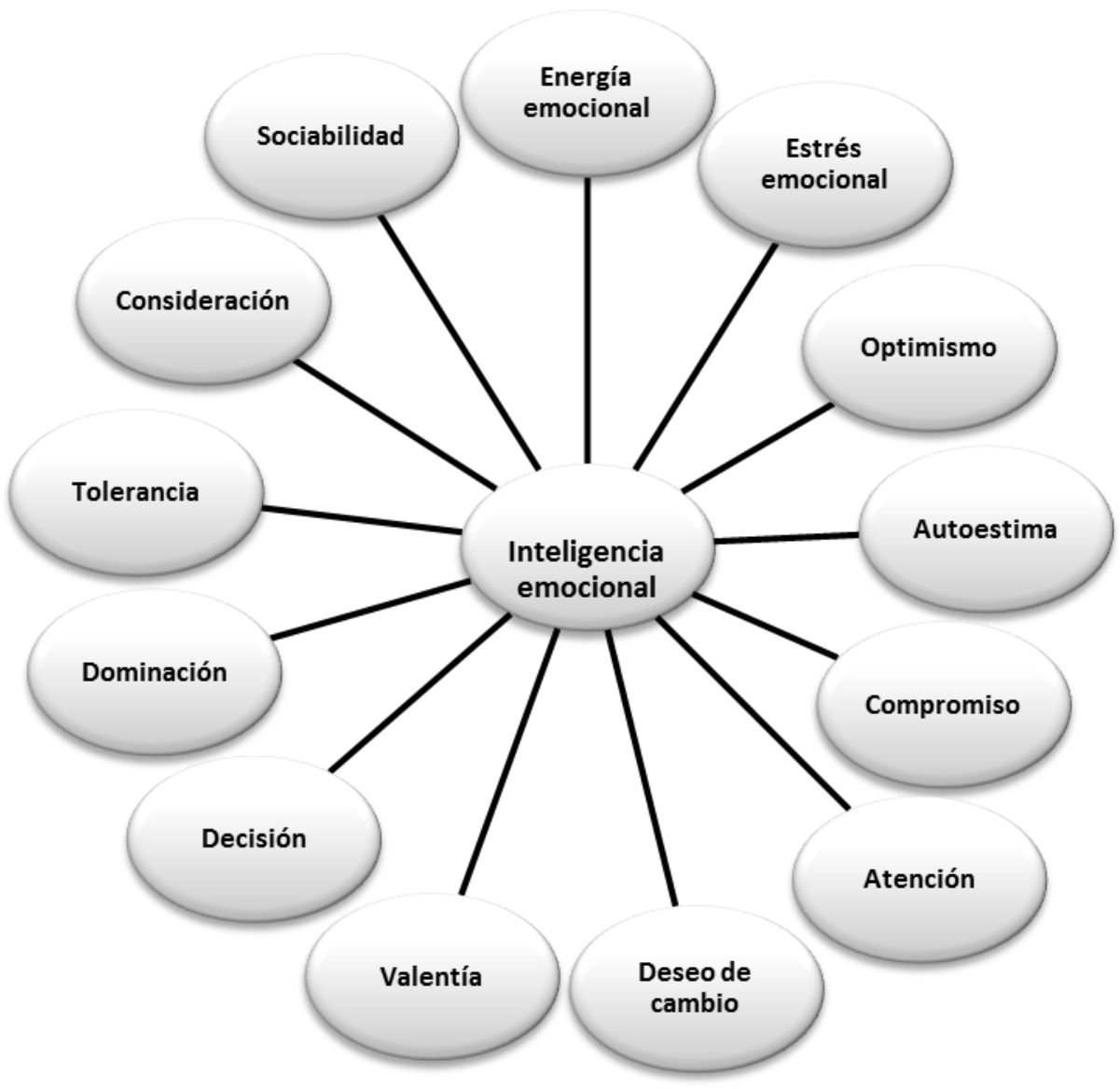

Fuente: Simmons y Simmos (1998)

Considerando la figura 1 es evidente que las habilidades emocionales son fundamentales en todos los niveles de organización social, las que pueden ser aplicadas por los padres en la crianza de los hijos, por lo docentes en su accionar profesional y en el ejercicio profesional en general, aquí lo fundamental es poder comprender los elementos que pueden influir en el estado anímico, sensación o sentimientos de los estudiantes para que se interesen por el aprendizaje de la temática que se aborda y también conociendo ello, se podrían promover interacciones en diferentes grados según las técnicas de enseñanza empleadas y mediadas por el docente. 
Experiencia e interacción de aula que se generan como ambiente de aprendizaje durante las clases de Biología. José Pereira-Chaves

Basado en lo descrito anteriormente se muestra un esquema en la siguiente figura sobre la interacción de las competencias emocionales propuestas por Bisquerra et al, (2009).

Figura 2. Interacciones de las competencias emocionales basado en Bisquerra et al., (2009)
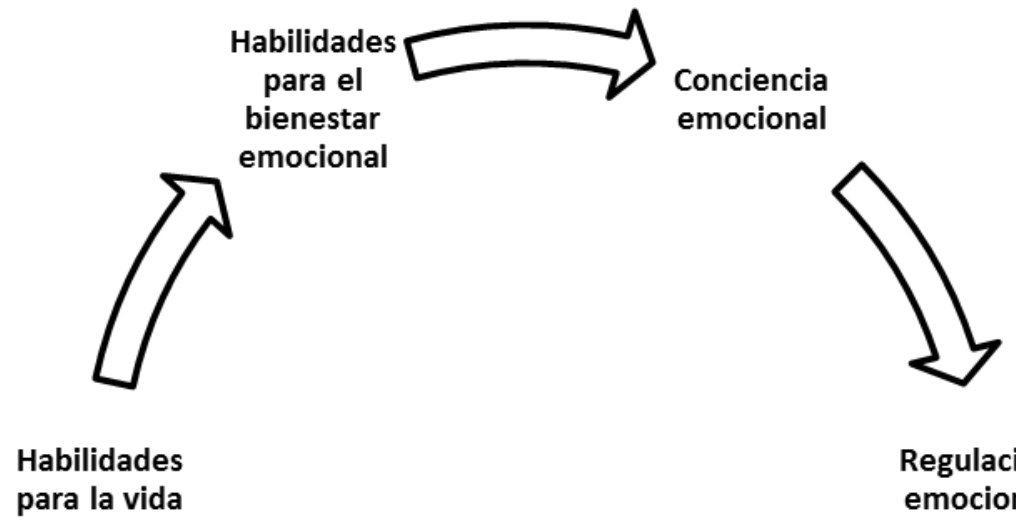

Regulación emocional
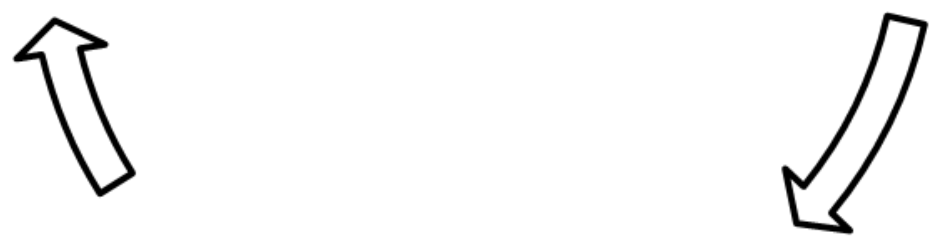

Habilidades socioemocionales

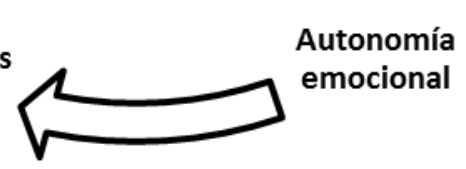

Fuente: Bisquerra et al., (2009)

Por lo que la capacidad para identificar, percibir e implementar las sensaciones, emociones y sentimientos en el aula a través de la comunicación verbal y no verbal es fundamental para que se desarrollen adecuadas interacciones y además el estímulo del interés hacia la adquisición de nuevos conocimientos en cada uno de los procesos 
mediáticos, donde se tome en cuenta las relaciones, e interés de los aprendices, para que puedan expresar sus sentimientos, emociones y afectos en momentos específicos. Es importante que el estudiante se sienta capaz de enfrentar con autonomía las vicisitudes que la misma le demanda, quien debe proceder estratégicamente aplicar lo que su profesor le ha enseñado y pueda ajustarlo a la exigencia para que pueda salir avante con su tarea, considerante que en las diversas actividades de aula e interacción el estudiante deberá ser capaz de argumentar, analizar y hacer representación que le permitan dar respuestas a situaciones desconocidas y que el profesor mediante su intervención pueda establecer diálogos que le permitan al estudiante apropiarse de esas habilidades que serán para la vida como lo es un individuo competente.

Para el trabajo de aula desde sus abordajes didácticos del contenido, el profesor debe establecer negociaciones y promover un ambiente en el aula que favorezcan las sinergias y trabajos colaborativo entre estudiantes, y tener una base sólida de cualidades propias de un buen facilitador y promotor de aprendizaje por ello Perrenoud (2007), señala que el docente debe dominar diez competencias: organizar y animar situaciones de aprendizaje, gestionar la progresión de los aprendizajes, elaborar y hacer evolucionar los dispositivos de diferenciación, implicar a los alumnos en sus aprendizajes y en su trabajo, trabajar en equipo, participar en la gestión de la escuela, informar e implicar a los padres, utilizar nuevas tecnologías, afrontar los deberes y dilemas éticos de la profesión y organizar la propia formación continua. Por otra parte, autores como García y Orozco (2008), Rueda (2009) y Luviano (2002) señalan que el profesor debe practicar la planeación del curso, la gestión de la progresión de los aprendizajes, la interacción didáctica en el aula y la evaluación de los aprendizajes, por ello se debe valorar el tipo de ambiente que se va promover porque de ello depende mucho que se promuevan actitudes a favor del aprendizaje.

En esta misma línea Pereira (2015) señaló elementos importantes en el proceso del aula como aporte a generar ambientes de interacción adecuada y promoción del conocimiento, en su estudio resalta el papel de los recursos didácticos y enfatiza el 
uso de las tecnología de la información y comunicación como ambiente de aprendizaje, por lo que invita a los profesores a usar las tecnología en su clase si el centro educativo cuenta con ellas, el docente debe de mediar el contenido y el aprendizaje de acuerdo con los objetivos cognitivos planteados, de tal manera, que los estudiantes se vuelven partícipes en su totalidad de los recursos accesibles y puedan establecer canales de comunicación e interacción que les permitan argumentar y aprender nuevos conceptos.

\section{Abordaje metodológico}

Para realizar la interpretación de la realidad educativa desde el punto de vista de las interacciones que se llevan a cabo en el aula como acciones que promueven el aprendizaje de los contenidos cognitivos, procedimientos y actitudes, se llevó a cabo una investigación durante el 2013, con el enfoque interpretativo cualitativo y el método etnográfico. Para ello se desarrolló un estudio de caso, en un Centro Educativo, ubicado en Heredia Costa Rica, con el único grupo de undécimo año, integrado por 24 estudiantes, de los que 12 eran hombres y 12 mujeres, el propósito fue analizar situaciones únicas, referentes a las interacciones o relaciones que se gestaban en el aula durante la mediación de los aprendizajes de las ciencias biológicas.

De acuerdo con Muñoz y Muñoz (2000), el objetivo básico del estudio de caso es llegar a la comprensión de la particularidad de éste, en el intento de conocer cómo funcionan las partes que lo componen y las relaciones entre ellas, para formar un todo. Para esta investigación fue necesario documentar la realidad del aula y conocer los aspectos propios en torno a la enseñanza y aprendizaje, los cuales se valoraron durante el desarrollo de este trabajo.

Se realizaron 18 observaciones participantes (OP) los días jueves de 8:00 a 10:15 am en las clases de biología desde febrero hasta setiembre del 2013, fecha que el grupo cerraba la programación oficial del currículo del programa de biología del ciclo diversificado, debido a que iniciaban las pruebas nacionales de bachillerato. Por otra 
parte se aplicaron 5 entrevistas semi-estructuradas a la profesora y 15 a estudiantes, en donde se contó con una guía de entrevista, en los cuales las preguntas estaban predeterminadas tanto en su secuencia como en su formulación para conocer los distintos momentos de interacción en el aula, además se trabajó con un grupo focal (Gf) conformado por seis estudiantes (3 hombre y tres mujeres).

Los datos se fueron agrupando en categorías de análisis según correspondía y haciendo la triangulación y las descripciones teóricas donde se evidenciara cada uno de las relaciones e interacciones desarrolladas en el aula.

\section{Principales Hallazgos}

El trabajo en el aula es clave para que se desarrollar el contenido de la mejor manera, y teniendo presente que para lograr un ambiente de comunicación asertiva la profesora tiene que ofrecer variadas alternativas, por lo que la diversidad de alternativas instruccionales en el aula es fundamental y los alumnos lo reclaman constantemente, aquí lo importante es aprovechar los recursos al máximo de acuerdo a la posibilidad que el centro educativo ofrezca, desde el uso de la tecnología que abarque nuevos métodos de estudio, hasta ingeniárselas con el uso tradicional de material didáctico, como una pizarra, de tal manera que logre interactuar con los alumnos de forma amena, responsable y comprometida.

A continuación se hace una descripción de lo observado en las clases, haciendo referencia al principio de las lecciones, considerando que el primer contacto con los estudiantes antes de comenzar una clase, podría favorecer a que el grupo esté dispuesto a seguir aprendiendo, y eso depende mucho de la actitud del docente, la proyección personal (Imagen) y la forma de saludarles e interesarse por sus alumnos.

[...] Al iniciar las lecciones la profesora, en su gran mayoría de las veces un(a) estudiante le ayuda a llevar el equipo audiovisual y materiales didácticos, y ella toma su tiempo para armar todo el equipo, los estudiantes esperan hasta que la profesora se 
acomode y mientras, hablan entre ellos. Una vez que está listo el equipo la profesora comienza con el saludo (Cuadro 1)

Cuadro 1. Resumen de las observaciones realizadas durante la entrada a las clases de biología.

- $\quad$ La profesora saluda a los estudiantes (sonríe), les dice que ya terminó el recreo, y que gusto estar con ellos de nuevo, que espera que hayan aprovechado su descanso. OP1

- La profesora, saluda y les dice a los alumnos que espera que la hayan pasado muy bien, sonríe y les dice a unos estudiantes que quería comer en clases que por ella no hay problema que coman galletas o jugos, pero que no pueden sacar el almuerzo en clases. OP2

- $\quad$ La profesora saluda a los estudiantes (sonríe), y les dice que espera todo les esté saliendo bien, y sobre todo en estos días que nuestras familias tengan trabajo y salud es la base de todo. OP4.

- La profesora saludas a los estudiantes, "Buenos días", se mostraba preocupada y pregunta por una alumna que había tenido un accidente e invita a los compañeros a que estén atentos de ella para que la apoyen. OP5.

- $\quad$ La profesora saluda a sus estudiantes y les dice que espera que les salga bien todo, les desea muchos éxitos en las diferentes elección de carreras universitarias, y que el examen de admisión es importante que lo tomen en serio, porque de ello depende que cumplan sus metas de entrar a carrera, les dice que no duda que les va ir bien porque ellos son excelentes estudiantes. OP15

Fuente: Elaboración propia.

Aquí es importante resaltar la preocupación de la profesora por saber cómo están los estudiantes, ella al entrar hace un reconocimiento visual a todo el grupo, les saluda con una sonrisa y sobre todo le proyecta a sus estudiantes, confianza, tranquilidad, lo que hace que haya disposición de trabajar; la actitud que tiene y proyecta un docente es fundamental para la interacción adecuada en el aula, que los estudiantes sientan la confianza y apoyo del docente y a la vez se genera esa interacción abierta, sincera y sin temores comunicativos para una pregunta y/o una respuesta, que se dé una 
interacción de igual a igual de respeto, trabajo, mediación y generación de conocimiento; así identificar de primera entrada lo que sucede y lo que hacen los alumnos, pueden favorecer a que se desarrollen los contenidos o tareas específicas de manera acertada y hasta motivante para ellos.

El docente tiene que tener claro su papel ante los adolescente en cuanto a su formación como las actitudes, valores y compromisos durante el desarrollo de su labor profesional y tener una adecuada comunicación pedagógica, Castellá et al (2007) señala que la comunicación es un aspecto transversal en el ámbito de aula, en donde el vínculo que utiliza el docente y los alumnos para entenderse, relacionarse y construir conocimiento es fundamental. Así el ambiente de aula condiciona en su gran mayoría el desarrollo de la enseñanza y aprendizaje, por lo que el logro del aprendizaje depende de la disponibilidad de los estudiantes y de la forma y visión conceptual, metodológica y actitudinal que tenga el docente de los contenidos disciplinares para la promoción de las competencias del pensamiento científico. Rué, 2010, resalta ..... "que en una situación de clase el profesor puede ser considerado un recurso de aprendizaje en la medida que atiende, presta su atención', proporciona apoyo afectivo, introduce un reto estimulante, ofrece referencia, informa, permite aclaraciones, entre otros" ( $p .17)$.

Con base en la opinión que tiene la profesora sobre la relación de profesor-alumno ella señala lo siguiente (Cuadro 2): 
Experiencia e interacción de aula que se generan como ambiente de aprendizaje durante las clases de Biología. José Pereira-Chaves

Cuadro 1. Opinión de la profesora sobre las relaciones que se llevan a cabo en el aula.

"La relación de profesor alumno es de igual-igual, abierta-dinámica y muy interactiva donde los estudiantes preguntan, cuestionan expresan libremente sus opiniones y experiencias cuando el tema es de su interés, cuando se da un ambiente de respeto y confianza, ellos tratan los temas de la misma forma, madurez de acuerdo a la edad, respetando las normas preestablecidas siempre busco crear un ambiente agradable en el aula donde se fomente valores de confianza, respeto, tolerancia, solidaridad y cooperación. Se hacen dinámicas para bajar la ansiedad cuando se percibe que están tensos o fatigados". EP1

"Muchas veces después de una dinámica, demuestran mucho más interés por la clase. Se observa que son más concentrados y poco distraídos, son alegres y amistosos. Esto facilita el proceso de aprendizaje, ya que se elimina un factor estresante (miedo al profesor) y el estudiante asume la materia como algo real, no como una demostración de un mundo abstracto". EP2

"Con la mayoría de estudiantes he podido generar relaciones de confianza donde el respeto mutuo es lo más importante y puedo profundizar los contenidos vistos en clase, muchos de ellos me participan en las olimpiadas de biología, porque son los que más aportan ideas y más cuestionamientos se hacen; pero siempre hay alguno por ahí que no tiene mucha empatía por la materia porque se trata de motivar, así el respeto y la formación de valores como la solidaridad, colaboración y el compañerismo siempre deben estar presentes". EP7.

"Con base en la interacción o relación de alumno-alumno es de respeto y comprensión, generalmente, compañeros más aventajados académicamente apoyan a los que menos comprenden. El ambiente global que se genera es muy favorable promueve la cooperación y facilita para que se dé el proceso de enseñanza-aprendizaje. Claro que en la relación e interacción de los estudiantes el docente debe ser un vigilante intermediario para fiscalizar y que su aprendizaje sea encausado". EP7.

Fuente: Elaboración propia.

Aquí se rescata la experiencia que tiene la profesora desde las distintas dimensiones de promoción del conocimiento, donde los alumnos jugaban un papel protagónico en el trabajo de aula, desde la organización individual y grupal que se desarrolla como espacio de trabajo e interacción; a la vez se llevan a cabo actividades de reflexión personal mediante el intercambio verbal donde se promueva la retroalimentación del proceso de enseñanza y aprendizaje. La interacción entre iguales influye en el desarrollo cognitivo lo que promueven interesantes debates e intercambios comunicativos del trabajo en equipo. 
Por otra parte, Prado et al (2010), señala que no basta con poner a los alumnos a trabajar juntos para que se produzcan efectos positivos en sus aprendizajes, sino que han de darse una serie de condiciones. Desarrollar los factores y variables que condicionan dicha efectividad supone rebasar los límites y objetivos del aprendizaje. No obstante, hay factores que destacan la eficacia del aprendizaje entre el que se destaca el trabajo cooperativo frente a las estructuras competitivas o individualistas. Así, las actividades de carácter cooperativo favorecen la aparición de conflictos cognitivos entre los aprendizajes, condición necesaria pero no indispensable para que se produzca un aprendizaje contextualizado y vivencial, además, proporcionan soporte o apoyo para resolver dichos conflictos.

Por lo que el trabajo en equipo no sólo incrementa el rendimiento de los estudiantes, sino que aumentan la motivación intrínseca y actitudes positivas hacia el aprendizaje, lo que corresponden a niveles altos de autoestima y favorecen la percepción positiva y el entendimiento con otros compañeros. Por otra parte la evaluación no siempre fue sumativa se generaba constantemente evaluaciones formativas, donde los estudiantes compartían lo que sabían y así una manera de socializar el nivel de logro y conocimiento que cada uno tenía. Por ello la docente siempre incentivaba en su trabajo, la criticidad y análisis mediante preguntas que provocaran la argumentación en el estudiantado.

Considerando lo citado en el párrafo anterior se rescata a Mercer (1997) quien cita que una buena reflexión para saber si se comprende bien un contenido, es tener que explicárselo a otra persona. $Y$ discutir de manera razonable con alguien al que puedas tratar como a un igual, social e intelectualmente es un método excelente para evaluar y revisar tu propia comprensión p. 99.

Así la profesora es una interlocutora que promueve la confianza y conocimiento mutuo de intereses personales e individuales de sus estudiantes, aunados a ello los roles institucionales que busca el desarrollo integral de todos los actores de interés, 
inmersos en el ambiente escolar, teniendo presente el enfoque con el que fue creado el centro educativo, se resalta el trabajo y el estímulo de la profesora en cada una de las actividades desarrolladas (Figura 3 )

Figura 3. Características que se generan producto de las interacciones entre la profesora de biología del Colegio basadas.

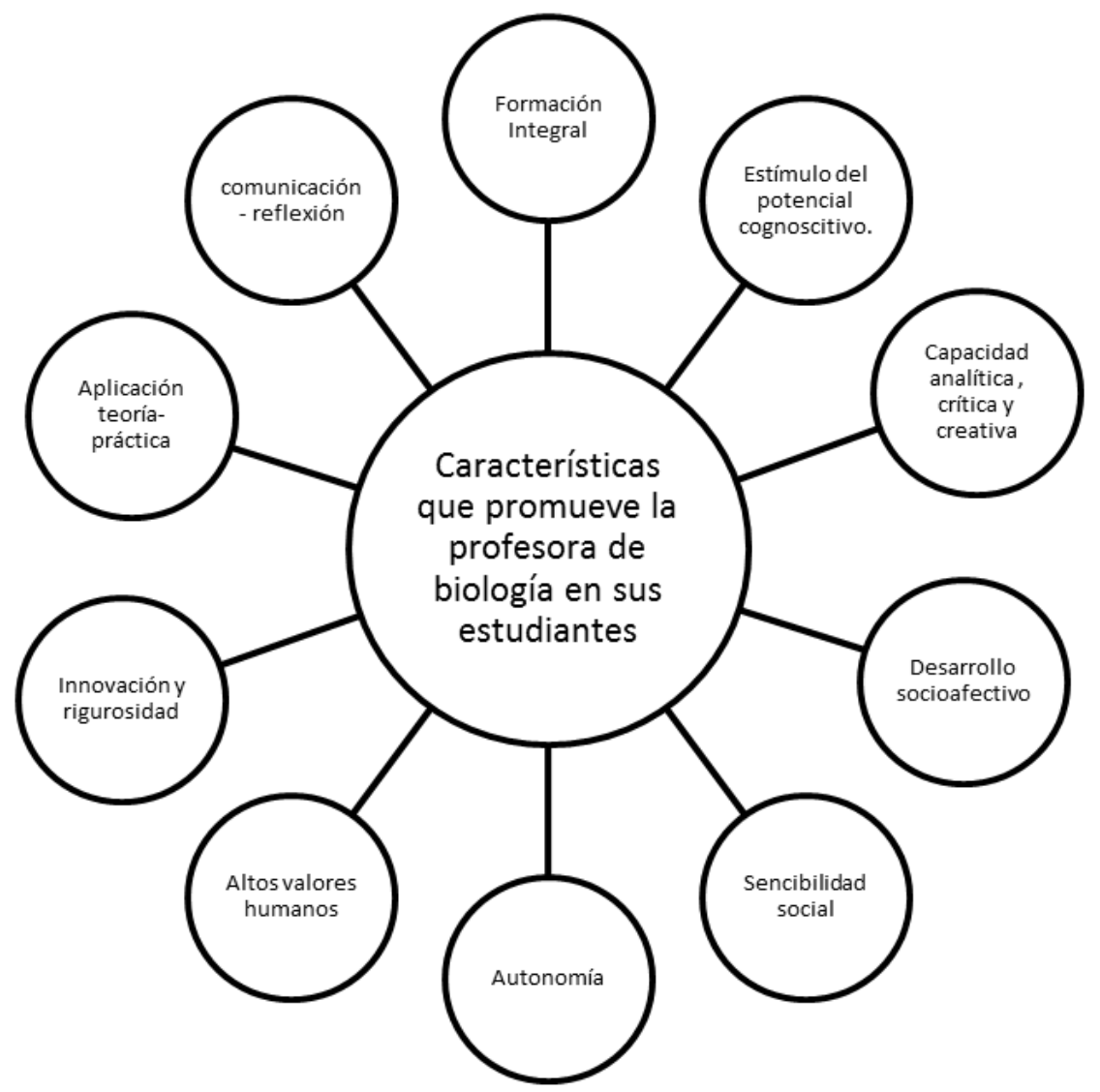

Fuente: Elaboración propia.

Aquí es importante resaltar que el personal académico y administrativo debe enfatizar en la reflexión y formación de valores fundamentales, como respuestas a los profundos desafíos éticos de la actualidad, donde se coloque al ser humano como un elemento fundamental, su pensamiento, cultura deben ser incorporadas en todas las disciplinas del saber, para el estímulo de la adquisición del conocimiento, así la interacción del 
aula debe ser adecuada, plena y motivadora para todos los participantes inmersos en el sistema y se generen sinergias de todo el proceso de enseñanza y aprendizaje, lo que se representa en la siguiente triangulación (Figura 4 ).

Figura 4. Proceso de interacción y aspectos que se consideran dentro de la mediación pedagógica en el aula según observaciones (Elaboración propia).

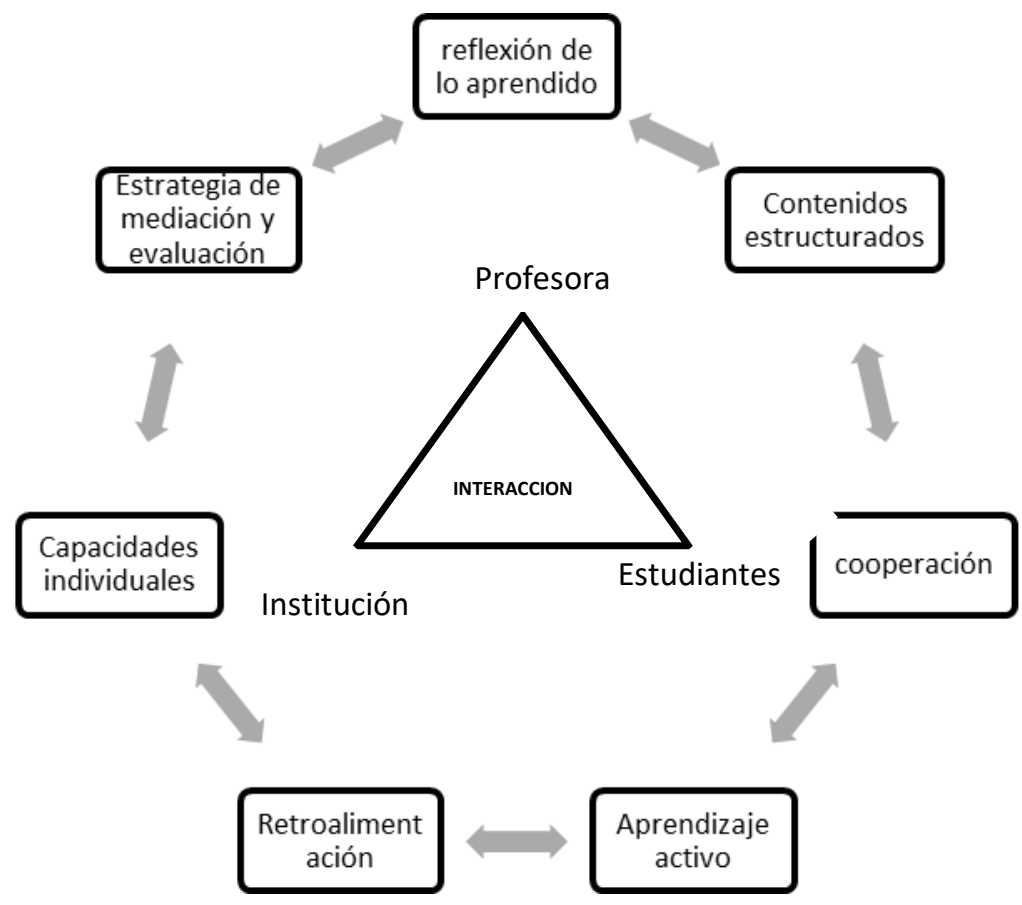

Fuente: Elaboración propia.

Aquí juega un papel fundamental la institución, debido a que es la responsable de promover en su personal académico, estudiantil y familiar, actitudes de compromiso, trabajo y dedicación, por ello la figura anterior hace alusión a esa interacción directa y gestora de una serie elementos que se promueven en el aprendizaje y formación de los estudiantes, hay directrices instituciones que resalta la actitud de los estudiantes, docentes y la mirada atenta de los familiares, para que se articulen acuerdos de compromiso que permita el adecuado y mutuo trabajo de construcción de conocimientos. Así la Institución como tal favorece y articula espacios académicos, extracurriculares e individuales donde se promueve la construcción de valores de 
solidaridad, respeto, trabajo en equipo, compromiso, liderazgo y criticidad no sólo en los estudiantes y su grupo de profesores, sino también entre las familias de cada uno de los alumnos, creando así las condiciones para la transformación de los espacios de aprendizaje promovidos en la institución.

Otro de los aspectos a resalta que se vivenció durante la investigación es la interesante interacción que se daba entre los estudiantes y la profesora sin importar la brecha generacional en ellos, considerando que la docente era una adulta mayor de más de 65 año, y los estudiantes de menos de 17 años, nunca de observó conductas reprochables de los alumnos hacia la profesora, sino más bien se generaba un ambiente de respeto y admiración, aspecto positivo que promueve el compromiso y responsabilidad de los estudiantes para con su profesora, así la docente en investigación, una profesional madura con la claridad del papel que cumple como formadora, líder, con autoridad escolar y vocacional.

Por otra parte haciendo una síntesis del trabajo con el grupo focal (GF) de estudiantes se obtuvo los siguientes resultados con base en las categorías de interacción profesoralumno-alumno:

Es una relación e interacción entre la profesora y nosotros súper buena, la profesora es increíble y muy querida, ella inspira mucha confianza lo que nos genera mucha tranquilidad en el aula, y se puede hablar de todo con ella, desde aspectos de la materia hasta cosas de relaciones amorosas y familiares, lo cual es bueno porque nos ayuda y motiva cuando lo requerimos. (Gf).

Aquí se puede interpretar que hay una participación de trabajo entre la profesora y los estudiantes que vinculan la actividad colegial, la práctica cotidiana desde una visión articulada en la formación integral que busca lograr la realización plena de los estudiantes. 
El interés que pone el estudiante sobre lo que aprende juega un papel determinante, y más aún cuando la materia no es su preferida hay que buscar la forma de poder motivarse y ejemplo claro es el siguiente párrafo:

La Biología no es mi materia favorita, pero lo que le puedo decir, es que a mí lo que me gusta es saber que la profesora me trata bien y podemos hablar de igual a igual, así en biología se lo que pasa en realidad, entender los procesos que ocurren en la vida, por ejemplo aplicarlo en uno mismo, entonces puedo entender mi metabolismo y muchas cosas más con una explicación del porque pasan las cosas. EE1.

Por otra parte los docentes tienen que prever las diversas interacciones que se estarán dando en el aula, por ello la claridad de la propuesta de las diversas actividades, donde la interacción de los estudiantes y la profesora según el objetivo de trabajo debe ser en el orden de una comunicación fluida, dinámica y sobre todo que se genere un espacio de aprendizaje mediante el logro de los objetivos de trabajo.

Se debe resaltar que los estudiantes tienen una visión y formación de lo que es el trabajo en equipo por la misma modalidad del colegio, aquí se generan espacios de interacción constantes en las diversas actividades curriculares y extracurriculares que presentan, como resultados de ello se evidencias las siguientes reacciones de estudiantes, según se muestra en la Figura 5: 
Experiencia e interacción de aula que se generan como ambiente de aprendizaje durante las clases de Biología. José Pereira-Chaves

Figura 5. Entrevista a los estudiantes sobre la relación que tiene con los compañeros.

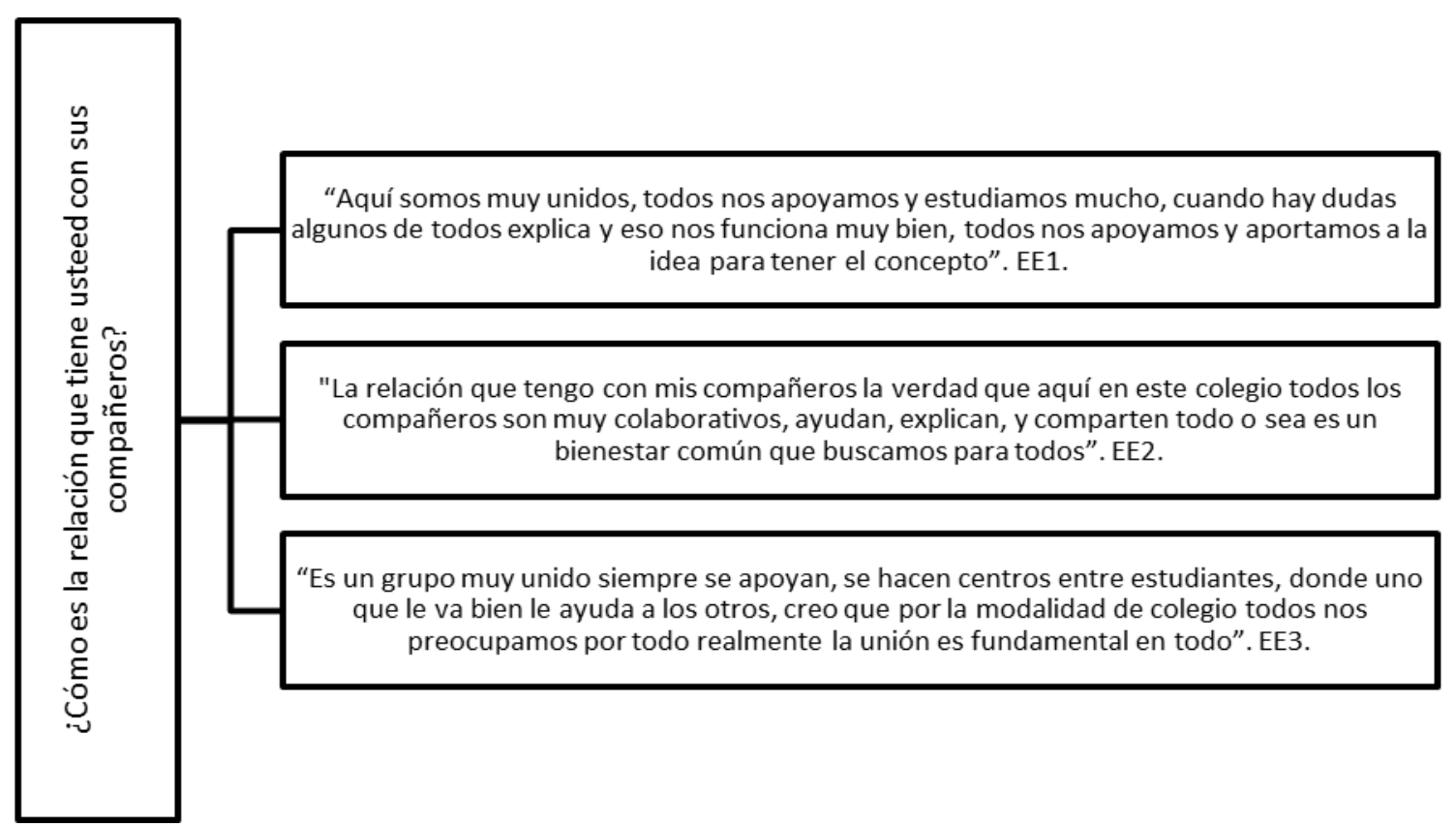

Fuente: Elaboración propia.

Por otro lado la imagen que tengan los estudiantes del docente es fundamental, porque eso favorece a que en el aula se den relaciones de respeto, compromiso y tratos de igual a igual tanto entre estudiantes como con la profesora, por lo que el concepto que se tenga del docente y la opinión que tienen nuestros estudiantes sobre las características personales es importante. Tener espacios de discusión, debate, y apertura de comunicación permanente en el aula como parte de una autoevaluación integral favorece el dinamismo y la sensibilidad en todos los niveles, aquí se destaca a Van Manen(2004) quien señala refiriéndose a tacto pedagógico que debe estar presente en el aula, donde se considera la autoridad pedagógica al docente, su competencia personal, profesional, su ética, la transposición didáctica y las formas de dominar la materia y tratar al grupo con sutileza, atino, respeto, logrando de la misma manera una respuesta respetuosa en todo sentido, siempre reconociendo el impacto de la educación en la conducta de todo ser humano, por lo que aquí juega un papel fundamental la vocación y la profesionalización para una adecuada gestión del 
Experiencia e interacción de aula que se generan como ambiente de aprendizaje durante las clases de Biología. José Pereira-Chaves

conocimiento en el aula. Por ejemplo los estudiantes tienen opiniones positivas de su profesora (figura 6):

Figura 6. Opinión de los estudiantes sobre la profesora de biología.

La profesora es muy simpática, explica muy bien y sobre todo lo comprende a uno, no es estricta sino inspira confianza para uno no fallarle, eso hace que se ponga mucha atención, por una situación de lealtad y respeto hacia ella. EE4.

La profesora es súper dulce, ella inspira mucha confianza y seguridad y sobre todo es una buena guía a pesar de su edad es muy especial, con nosotros. EE7.

La profesora siempre nos trata con mucho amor a todos, es muy dulce y eso lo motiva a uno a estudiar y a no faltar a las clases es imposible faltar, porque es como faltarle el respeto a ella de lo buena gente que es y por su puesto el rol de nosotros ha sido de almiararla y quererla mucho. EE11.

Es muy responsable, respetuosa, lo primero que todo nos ha enseñado que primero está la humanidad, nosotros la respetamos y la queremos mucho, realmente inspira ser responsable, aunque no lo seamos en otras materias con ella sí lo somos. EE8.

La profesora se desempeña muy bien en el aula, porque nos ha explicado bien la materia y hemos aprendido bastante durante estos dos años, es muy simpática, amable, comprometida, proyecta confianza y sobre todo nos quiere. EE10.

Fuente: Elaboración propia.

Lo anterior pone en evidencia las características propias de la docente donde la personalidad, la comunicación y su formación académica juega un papel primordial en la confianza y la percepción positiva que los estudiantes tienen de ella, lo que concuerda muy bien con los datos obtenidos durante la observación en donde se registraron sus acciones en el aula, y se resaltan algunos de los datos donde no sólo se considera la personalidad sino que es una amalgama de personalidad y estrategias didácticas, a la vez ella proyecta a sus estudiantes cuando desarrolla la temática la pasión y el amor de lo que enseña, vive, siente y ama realmente la profesión docente y sobre todo el ser una educadora con un alto contenido disciplinar en su carrera de bióloga, los siguientes tres registros durante la observación participante (OP) muestran principalmente las estrategias abordadas, y en ellas se reflejaban el sentido de la profesora por los estudiantes. 
La profesora, toma una revista, hace preguntas sobre entomología y luego comienza a explicar a sus estudiantes articulando así lo que señalan los estudiantes para la explicación de las características de las plantas insectívoras que son pobres en nitratos y la forma de obtener el nitrógeno es por medio del insecto, que tiene varias estrategias que tiene estructuras táctiles. OP 11.

La profesora hace algunas preguntas a los estudiantes sobre lo que saben de hacer resúmenes, esquemas, mapas conceptuales una vez discutido sobre ello, comienza a explicarles a los estudiantes cómo elaborar un mapa conceptual, y les da los criterios de elaboración. Los estudiantes salen a trabajar en grupos de 4 fuera del aula en unas bancas, en el comedor y el jardín. OP8

La profesora comienza a desarrollar el tema protista en la pizarra y los caracteriza, usa palabras de increíbles, interesantes, que son beneficiosos, pero también generan muchas enfermedades, lo que utiliza para hacerles preguntas a sus estudiantes sobre ¿cuáles creen ustedes que serían esas enfermedades? OP6.

Las frases anteriores parecen una descripción de técnicas utilizadas en la clase, pero aquí más que una forma de presentar el contenido es la afectividad y las buenas relaciones que se dan en el aula entre la docente y los estudiantes, y en las siguientes opiniones que tienen los estudiantes sobre el papel que cumple la profesora en la clase se tiene más claridad (figura 7): 
Experiencia e interacción de aula que se generan como ambiente de aprendizaje durante las clases de Biología. José Pereira-Chaves

Figura 7. Opinión que tienen los estudiantes sobre el papel que ha jugado la profesora.

El papel que ha jugado la profesora es excelente ya que gracias a ella nosotros hemos aprendido lo que hasta ahora sabemos y además es súper comprometida y le encanta lo que hace que es enseñarnos biología, entonces por esto es que ambos hemos jugado un papel muy importante para nuestro futuro. EE9.
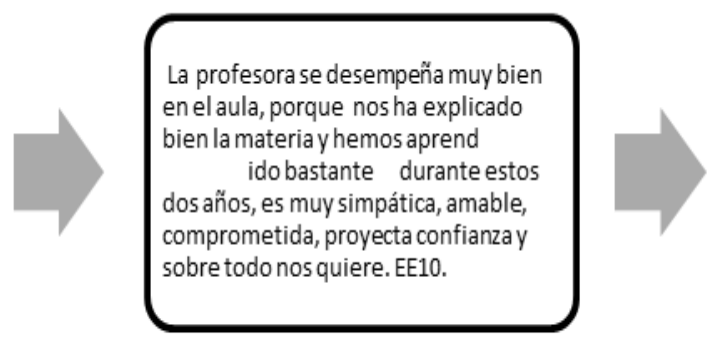

El papel de la profesora es enseñarnos, y sobre todo ella sabe hacerlo muy bien, a pesar que es una señora mayor es muy ubicada con los estudiantes, es muy buena gente. EE5.

Ha jugado un papel muy importante porque gracias a ella he aprendido muchas cosas del temay sobre todo a valorar todo lo que está alrededorya leer todas las noticias impresas sobre biología nos ha creado la cultura de la exploración. EE12.
El papel de la profesora es guiarnosy sobre todo es muy simpática difícil de no querer, es muy dinámica, abierta al dialogo y sobre todo comprometido con nosotros los estudiantes, ella nos quiere mucho. EE6.

Fuente: Elaboración propia.

Como forma de mantener la motivación del estudiantado la profesora durante la clase utilizaba con frecuencia expresiones como: ¡muy bien! ¡Excelente!, me parece muy interesante su observación (OP1...16), aspectos que son positivos a la hora del desarrollo de la clase, porque los estudiantes no sólo están atentos a participar sino a realizarlo de la mejor manera. La profesora organiza sus interacciones con los estudiantes por medio de preguntas enfocadas a las temáticas del curso, las cuales están dirigidas a promover el aprendizaje del contenido y a mantener la atención del grupo a partir de un reconocimiento de la relevancia del contenido.

Por otra parte, es común que después de una intervención del estudiante o una exposición, la profesora retroalimente el aporte con una representación gráfica de 
esquemas que visibilicen el contenido a comprender, como figuras de los libros de texto universitarios y de material didáctico en línea especializado.

Durante el periodo de observación de lecciones se evidenció el uso de distintas estrategias didácticas de comunicación (tono de voz, deslizamiento de mirada), socialización (interacción activa en clases, comparte muchas experiencia de ella con los alumnos y viceversa) y mediación (las variadas técnicas para el desarrollo del contenido) del aprendizaje, abordándose así diversidad de posibilidades de potencializar las distintas habilidades de los estudiantes al ser partícipes de alguna de todas las estrategias utilizadas.

El planteamiento que propone la profesora en su secuencia didáctica y metodológica, permite apreciar la relación interactiva de la profesora-estudiantes-conocimiento, el desarrollo de estilos personales y se promueven las metodologías de construcción de los conocimientos. Aquí el papel de la docente es de guía del proceso de aprendizaje, donde a través de la observación en el aula se logra interpretar que muchas veces la docente controla, por medio de la observación (deslizamiento de su mirada), a sus estudiantes en cuanto al desempeño o trabajo de los mismos, generando inquietudes en ellos, reflexionando en torno a errores o conceptos mal empleados, promoviendo el debate, la discusión e interacción general del grupo para el desarrollo y construcción de significados.

Por lo que la articulación de las distintas estrategias observadas en las clases promueve en los estudiantes interacciones activas y de colaboración en donde se estimula colaborativamente la profundización conceptual y el desarrollo de competencias del pensamiento científico a través de preguntas generadoras.

La relación entre enseñanza y aprendizaje implica un abordaje de estrecha interacción entre el profesor y los estudiantes, en donde constantemente se generan espacios de reflexión entre ambos actores, el aula es un sitio de aprendizaje continuo que no debe 
ser visto únicamente limitado a procesos cognitivos, sino que va más allá de ello, incluye la parte emocional, social, afectiva, psicológica, cultural y otras condiciones que surgen producto de la dinámica y del trabajo en torno al proceso de enseñanzaaprendizaje.

Por otra parte el aula debe ser espacios comunicativos para el cumplimiento de los propósitos de aprendizaje, y las clases dentro de ellas deben ser dinámicas, estructuradas y flexibles, donde el planeamiento responda a lo que el profesor promueva aprender, el cómo se quiere abordar, y lo que se desea lograr. En las lecciones se gestan negociaciones entre el docente y los estudiantes que conlleva a reorganizaciones constantes, y su calidad, riqueza y fluidez van a depender de la comprensión y la capacidad de ajustarse a las normas que rigen este tipo específico de intercambio comunicativo que se genera en la clase.

Durante las clases se llevan a cabo diálogos muy productivos que circulan entorno a la temática que se está desarrollando pero en ocasiones la misma divergencia de interés de los participantes, puede que la concentración y sus intereses estén desarrollando diálogos paralelos entre partes que no son propios del contenido en discusión de la clases, sino más bien una vivencia social ( quién puede hablar, a quién, cuándo, de qué manera, sobre qué, para qué), así se desarrolla el contenido académico donde se abordan los temas, se asignan obligaciones y responsabilidades de las partes. Por ello un profesor que planifica y prepara sus clases lo más cautivadora para los estudiantes debe incluir la manera de cómo éstos responden a sus obligaciones y demandas de las tareas propias de su quehacer estudiantil, de manera que se logre la atención de todos y todas.

Así el aula se convierte en todo un desarrollo de contextos individuales, grupales, cooperativo donde el compromiso de estudiantes y profesor debe ser uno, que es la adquisición de conocimiento de una manera fluida y dinámica sea con la presencia del profesor o con las explicaciones magistrales de éste. Así hablamos que el 
conocimiento en el aula se lleva a cabo con la interacción directa del profesor y sus alumnos, y de los alumnos entre ellos, cada hecho o relación en el aula tiene su propio hilo conductor, en donde incluye actividades que tienen su propio espacio, el cual tomar en cuenta que los estudiantes son partícipes de lo que se planea en el aula, y son los responsable por igual de la dinámica que se dé a la hora del desarrollo conceptual, operacional, metodológico, actitudinal de lo que se enseña y aprende.

\section{Reflexiones Conclusivas}

Las clases de biología se desarrollaron bajo diálogos muy productivos que circulaban entorno a la temática desarrollada, los alumnos jugaban un papel protagónico en el trabajo de aula desde la organización individual y grupal como espacio de trabajo e interacción; se desarrollaron actividades de reflexión individual mediante el intercambio verbal, donde se promovía la retroalimentación del proceso de enseñanza y aprendizaje.

La docente jugó un papel fundamental debido a que se identificó con su grupo de estudiantes, en todo momento promovía la partición y uso de las distintas habilidades cognitivas, donde se puso en juego la sensación, intuición, pensamiento, sentimiento de los estudiantes, independientemente de sus características: extrovertidos quienes eran emotivos y expresivos, mientras que a la vez los introvertidos eran más reflexivos y reservados en sus emociones y manera de interactuar.

Se evidenció la tendencia de la profesora de identificarse con el paradigma constructivista, buscando durante el desarrollo del contenido motivar al estudiante a que se plantee situaciones que lo hagan entender los conceptos y plantearse soluciones reales dentro de sus esquemas mentales que poseen, la profesora fue una interlocutora donde siempre promovió la confianza y conocimiento mutuo de intereses personales e individuales de sus estudiantes. 
La institución juega un papel fundamental en la formación de valores, debido a que es en gran medida la responsable de promover en su personal académico y estudiantil, actitudes de compromiso, trabajo y dedicación, por ello la interacción directa y gestora que se promueven en el aprendizaje y formación de los estudiantes son primordiales, y eso es promovido por las directrices instituciones que resalta la actitud de los estudiantes, docentes y la mirada atenta de los familiares, donde se articulan acuerdos de compromiso que permitan el adecuado y mutuo trabajo de construcción de conocimientos.

El docente debe tener una comunicación asertiva con sus estudiantes y promover espacios de interacción de cordialidad, respeto y convivencia permanente donde se gesten actitudes positivas hacia el aprendizaje.

\section{Referencias}

Bisquerra, R., Pérez, N., Cuadrado, M., López, E., Filella, G. y Obiols, M. (2009). Actividades para el desarrollo de la inteligencia emocional en los niños. España: Parramón Ediciones.

Blandón, M., Molina, V. y Vergara, E. (2006). Los estilos directivos y la violencia escolar. Las prácticas de la educación física. Revista Iberoamericana de Educación, 38, 87-103.

Castellá, J., Comelles, S., Cros, A. y Vilá, M. (2007). Entender (se) en clase. Las estrategias comunicativas de los docentes bien valorados. Barcelona España: Editorial Graó.

Estrada, R. y Monroy, S. (2002). Perspectivas múltiples, multi-paradigmas y participación sistémica en las organizaciones. Memoria en CD 6a Congreso Nacional y 1a Internacional de Investigación: Paradigmas Emergentes de la Administración en las Sociedades del Conocimiento, organizado por la Academia de las Ciencias Administrativas ACACIA y por la Escuela Superior de Comercio y Administración ESCA, del Instituto Politécnico Nacional IPN, México, Abril.

Extremera, N. y Fernández-Berrocal, P. (2004a).La importancia de desarrollar la inteligencia emocional en el profesorado. Revista Iberoamericana de Educación, 33(8). 
García, M. y Orozco, L. (2008). Orientando un cambio de actitud hacia las ciencias naturales y su enseñanza en profesores de educación primaria. Revista Electrónica de Enseñanza de las Ciencias, 7 (3), 539-568.

Hilera, J. y Palomar, D. (2008). Modelado de procesos de enseñanza-aprendizaje reutilizables con XML, UML e IMS-LD. Revista Educación a Distancia, número monográfico II.

Luviano, J. D. (2002). Marco conceptual y metodológico para evaluar el desempeño docente en las maestrías del CENIDET. Centro Nacional de Investigación y Desarrollo Tecnológico: p.8.

Mercer, N. (1997). La construcción guiada del conocimiento: el habla de profesores y alumnos. Barcelona: Paidós.

Muñoz, P. y Muñoz, I. (2000). Intervención en la familia: Estudio de casos. En G. Pérez (Coord.), Modelos de investigación cualitativa en educación social y animación sociocultural (pp 221-252). Aplicaciones prácticas. Madrid: Narcea Ediciones.

Pereira, J. (2015). Las estrategias metodológicas en el aprendizaje de la biología. Uniciencia, 29(1), 58-81.

Perrenoud, P. (2007). Utilizar las nuevas tecnologías. En Diez nuevas competencias para enseñar, (5⿳⺈冂䒑 ed.), pp.107-120. Barcelona: Grao.

Prado, J. C., García, J., Mejías, A. y Fernández, A.F. (2010). Desencadenantes, resultados y factores críticos de éxito en los sistemas de participación del personal. Resultados de un estudio en España. Dirección y Organización. №. 42, pp. 71-82.

Rué, J. (2010). Entre la tradición y el hiperactivismo. Cuadernos de Pedagogía no 403. Monográfico julio-agosto.

Rueda, M. (2009). La evaluación del desempeño del docente: consideraciones desde el enfoque por competencias. Revista Electrónica de Investigación Educativa. 11(2), $1-16$.

Simmons, S. y Simmons, J. (1998). E.Q. Como medir la Inteligencia Emocional. España, Editorial EDAF S.A. Madrid, España.

Van Manen, M. (2004). El tono en la enseñanza: El lenguaje de la pedagogía. Edición en castellano: Barcelona: Paidós. 